\title{
Correction to: Improved Test Solutions for COTS-Based Systems in Space Applications
}

\author{
Riccardo Cantoro, Sara Carbonara, Andrea Floridia, Ernesto Sanchez, \\ Matteo Sonza Reorda, and Jan-Gerd Mess
}

\section{Correction to: \\ Chapter "Improved Test Solutions for COTS-Based Systems in Space Applications" in: N. Bombieri et al. (Eds.): VLSI-SoC: Design and Engineering of Electronics Systems Based on New Computing Paradigms, IFIP AICT 561, https://doi.org/10.1007/978-3-030-23425-6_10}

The original version of this chapter contained an error in the third author's name. The spelling of Andrea Floridia's name was incorrect in the header of the paper. The author's name has been corrected. 